\title{
POLYCYCLIC AROMATIC HYDROCARBONS AS STAR FORMATION RATE INDICATORS
}

\author{
D. Calzetti ${ }^{1}$
}

\begin{abstract}
As images and spectra from ISO and Spitzer have provided increasingly higher-fidelity representations of the mid-infrared (MIR) and Polycyclic Aromatic Hydrocarbon ( $\mathrm{PAH}$ ) emission from galaxies and galactic and extra-galactic regions, more systematic efforts have been devoted to establishing whether the emission in this wavelength region can be used as a reliable star formation rate indicator. This has also been in response to the extensive surveys of distant galaxies that have accumulated during the cold phase of the Spitzer Space Telescope. Results so far have been somewhat contradictory, reflecting the complex nature of the PAHs and of the mid-infrared-emitting dust in general. The two main problems faced when attempting to define a star formation rate indicator based on the mid-infrared emission from galaxies and star-forming regions are: (1) the strong dependence of the PAH emission on metallicity; (2) the heating of the PAH dust by evolved stellar populations unrelated to the current star formation. I review the status of the field, with a specific focus on these two problems, and will try to quantify the impact of each on calibrations of the mid-infrared emission as a star formation rate indicator.
\end{abstract}

\section{Introduction}

First enabled by the data of the Infrared Space Observatory (ISO, Kessler et al. 1996) and then expanded by the images and spectra of the Spitzer Space Telescope (Spitzer, Werner et al. 2004), the mid-infrared, $\sim 3-40 \mu \mathrm{m}$, wavelength range has been at the center of many investigations seeking to define handy and easy-to-use Star Formation Rate (SFR) indicators, especially in the context of extragalactic research.

In the Spitzer (cold-phase) era, the MIR emission from galaxies had experienced renewed interest particularly in the high-redshift galaxy populations community (e.g., Daddi et al. 2005,2007; Papovich et al. 2006,2007; Yan et al. 2007;

\footnotetext{
1 Dept. of Astronomy, University of Massachusetts, Amherst, MA 01003, USA, e-mail: calzetti@astro.umass.edu
} 
Reddy et al. 2010). The Spitzer Multiband Imaging Photometer (MIPS, Rieke et al. 2004) and the Spitzer InfraRed Spectrograph (IRS, Houck et al. 2004) had proven particularly sensitive at detecting the MIR dust emission from galaxies in the redshift range $\mathrm{z} \approx 1-3$. Observations performed with the MIPS $24 \mu \mathrm{m}$ and $70 \mu \mathrm{m}$ bands yield the restframe $\sim 8 \mu \mathrm{m}$ and $23 \mu \mathrm{m}$ emission of a $\mathrm{z}=2$ galaxy. An obvious question to ask is whether, and to what degree, the MIR emission traces the recent star formation in galaxies.

The MIR region hosts the rich spectrum of the Polycyclic Aromatic Hydrocarbon (PAH) emission features in the wavelength range 3-20 $\mu \mathrm{m}$ (Léger \& Puget 1984), also previously known as Unidentified Infrared Bands or Aromatic Features in Emission (e.g., Sellgren et al. 1983; Sellgren 1984; Puget et al. 1985), which are the subject of the present Conference. Thus, a corollary to the question in the previous paragraph is how well the PAH emission traces recent star formation in galaxies.

In this Review, I concentrate on results obtained from the investigation of nearby (closer than about 30-50 Mpc) galaxies, where the MIR emission can be typically resolved to sub-kpc scale at the Spitzer resolution in the $3-8 \mu \mathrm{m}$ range: the $2^{\prime \prime}$ FWHM corresponds to $\sim 0.5 \mathrm{kpc}$ for a galaxy at $50 \mathrm{Mpc}$ distance. In this regime, separating the contributing heating effects of various stellar populations become easier than in unresolved galaxies. Throughout this paper, I will refer to "8 $\mu \mathrm{m}$ emission" when indicating the stellar-continuum subtracted emission as detected by Spitzer in the IRAC $8 \mu \mathrm{m}$ band (Fazio et al. 2004) or similarwavelength bands on the ISO satellite. With the terminology "PAH emission" I will refer to the emission features (after subtraction of both the stellar and dust continuum) at/around $8 \mu \mathrm{m}$.

An important assumption when using the infrared emission as a SFR indicator is that galaxies must contain sufficient dust that a significant fraction of the UV-optical light from recently formed stars is absorbed and re-emitted at longer wavelengths by the dust itself. For the "typical" galaxy in the Universe, about half of its energy budget is re-processed by dust in the infrared (e.g., Hauser \& Dwek 2001; Dole et al. 2006), but a very large scatter on this "mean" value exists from galaxy to galaxy. There tends to be a relation between star formation activity and dust opacity in galaxies, in the sense that more active galaxies also tend to be more opaque (e.g., Wang \& Heckman 1996; Heckman et al. 1998; Calzetti 2001; Sullivan et al. 2001). In general, infrared SFR calibrations will need to take into account that a fraction of the star formation will emerge from the galaxy unprocessed by dust, and this fraction will depend on a number of factors (metal content and star formation activity being two of those, and dust geometry being a third, harder-to-quantify, factor).

\section{PAH emission as a SFR indicator}

The $8 \mu \mathrm{m}$ emission represents about $5 \%-20 \%$ of the total infrared (TIR) emission from galaxies; of this, more than half, and typically $70 \%$, can be attributed to PAH emission in metal-rich galaxies (Smith et al. 2007; Dale et al. 2009; 
Marble et al. 2010). In this context, "metal-rich" refers to oxygen abundances larger than $12+\log (\mathrm{O} / \mathrm{H}) \sim 8.1-8.2$ (with the Sun's oxygen abundance being $~ 8.7$, Asplund et al. 2009). Although small, the fraction of TIR energy contained in the $7-8 \mu \mathrm{m}$ wavelength region is not entirely negligible, and much effort has been devoted to investigating whether the emission in this region could be used as a reliable SFR indicator.

Systematic efforts began with ISO (e.g., Roussel et al. 2001; Boselli et al. 2004; Forster-Schreiber et al. 2004; Peeters et al. 2004), and continued with Spitzer, which enabled extending the analysis to fainter systems and to higher spatial detail (e.g., Wu et al. 2005; Calzetti et al. 2005, 2007; Alonso-Herrero et al 2006; Zhu et al. 2008; Kennicutt et al. 2009; Salim et al. 2009; Lawton et al. 2010). In a typical approach, the $8 \mu \mathrm{m}$ (or similar wavelengths) luminosity or luminosity/area is plotted as a function of other known SFR indicators, to establish whether a correlation exists; an example is shown in Figure 1. In one case, the $8 \mu \mathrm{m}$ emission has also been combined with tracers of the unattenuated star formation (e.g., $\mathrm{H} \alpha$ line emission) to recover an "unbiased" SFR indicator (Kennicutt et al. 2009).

Most of these analyses recover a linear (in log-log space) relation with slope of unity, or slightly less than 1 , between the $8 \mu \mathrm{m}$ emission and the reference SFR indicator. A roughly linear trend with unity slope would in general indicate that the $8 \mu \mathrm{m}$ luminosity is an excellent SFR tracer; however, as we will see in the next two sections, the PAH emission (and, therefore, the $8 \mu \mathrm{m}$ emission, of which the PAH emission represents typically more than half of the total) shows a strong dependence on the metal content of the region or galaxy, and a less strong, but possibly significant, dependence on the nature of the heating stellar population.

\section{The dependence of PAHs on metallicity}

Already known for more than a decade (e.g., Madden 2000, and references therein), the dependence of the intensity of the PAH emission on the galaxy/environment metallicity has been quantified in detail by Spitzer data (e.g., Fig. 1). This dependency is on top of the general decrease of the TIR intensity for decreasing metallicity, i.e. the decrease in overall dust content. Analyses of galaxy samples and of galaxy radial profiles covering a range of metallicity show an additional order-of-magnitude decrease in the $8 \mu \mathrm{m}$-to-TIR luminosity for a factor $\sim 10$ decrease in metallicity, with a transition at $12+\log (\mathrm{O} / \mathrm{H}) \approx 8.1$ (Boselli et al. 2004; Madden et al. 2006; Engelbracht et al. 2005; Hogg et al. 2005; Galliano et al. 2005,2008; Rosenberg et al. 2006; Wu et al. 2006; Draine et al. 2007; Engelbracht et al. 2008; Gordon et al. 2008; Muñoz-Mateos et al. 2009; Marble et al. 2010).

MIR spectroscopy with Spitzer has established that the observed low abundance of PAHs in low metallicity systems is not due to the molecules being more highly ionized and/or dehydrogenated than in higher metallicity galaxies (Smith et al. 2007), although there is controversy on whether they could be characterized by smaller or larger sizes (Hunt et al. 2010; Sandstrom et al. 2010; see, also, 


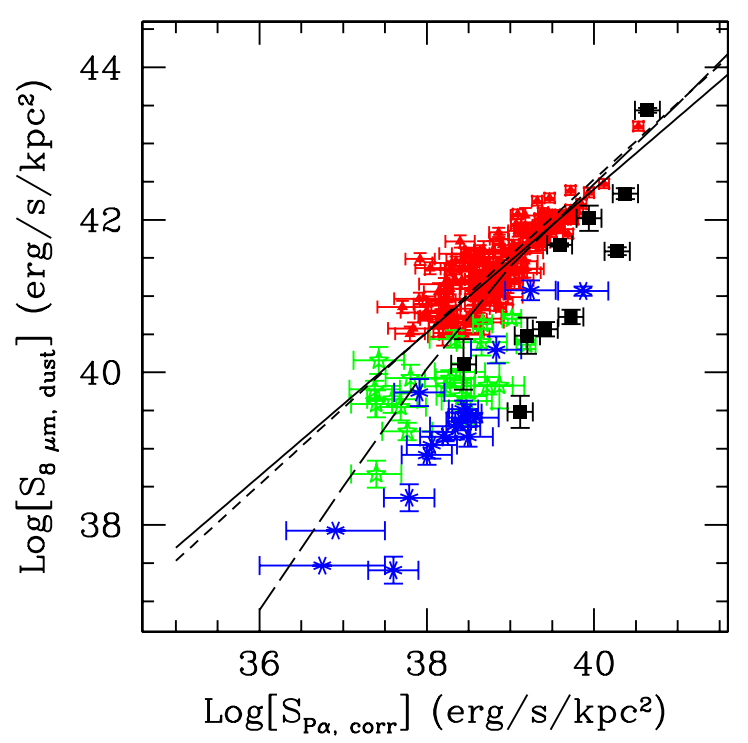

Fig. 1. The surface luminosity density at $8 \mu \mathrm{m}$ (stellar-continuum subtracted) as a function of the surface luminosity density of the extinction-corrected hydrogen recombination emission line $\mathrm{P} \alpha(1.8756 \mu \mathrm{m})$, for HII knots (red, green, and blue symbols) and low-metallicity starburst galaxies (black symbols). The HII-knots are typically $\approx 0.5 \mathrm{kpc}-$ size line-emitting regions from 33 nearby galaxies; there are a total of 220 regions in the plot. The starburst galaxies are from the sample of Engelbracht et al. (2005). The red symbols show regions hosted in galaxies with oxygen abundance $12+\log (\mathrm{O} / \mathrm{H})>8.35$. Regions of lower metal abundance are divided between those with oxygen abundance $8.00<12+\log (\mathrm{O} / \mathrm{H}) \leq 8.35$ (green symbols) and those with $12+\log (\mathrm{O} / \mathrm{H}) \leq 8.00$ (blue symbols). The continuous line shows the best linear fit (in the log-log plane) through the high metallicity data points (red symbols). The dash line shows the best linear fit with unity slope. A common result is for the best fit to yield a slope lower than unity. The dot-dash curve shows the locus of a model for which regions/galaxies suffer from decreasing dust attenuation (and, consequently, decreasing dust emission) as their total luminosity decreases, a commonly observed trend in galaxies and star-forming regions (see references in the last paragraph of the Introduction). Even when taking this effect into account, low metallicity regions and starburst galaxies display a depressed $8 \mu \mathrm{m}$ emission, which has been shown to be due to deficiency of PAH emission. From Calzetti et al. (2007).

Hunt et al. elsewhere in this volume and Sandstrom et al. elsewhere in this volume).

The nature of the correlation between the strength of the PAH emission features and the region's metallicity is still ground for debate. The two main scenarios 
that can account for the observed trend are: the "nature" scenario, according to which lower metallicity systems have a delayed PAH formation, and the "nurture" scenario, according to which PAHs are processed and/or destroyed in the harder radiation fields of low metallicity galaxies.

Galliano et al. (2008, see, also, Galliano elsewhere in this volume) suggest that the PAH strength-metallicity correlation may be due to the delayed formation of the PAHs themselves, which are thought to form in the envelopes of carbon-rich AGB stars. In a young system, the first dust will emerge from supernovae (timescale $<10 \mathrm{Myr}$ ), while AGB-produced dust will emerge at a later stage (timescale $\approx 1$ Gyr). This scenario may be difficult to reconcile with the fact that low-metallicity systems in the local Universe contain stellar populations that are typically older than 2 Gyr (Tosi 2009 and references therein), and have median birth parameters that are not drastically different from those of more metal rich galaxies (Lee et al. 2007). However, low-mass systems are also thought to lose most of their metals during the sporadic events of star formation that characterize their typical star formation history (e.g., Romano et al. 2006). Clearly, self-consistent models for the metal enrichment of dwarf, low-metallicity galaxies that also include their extended star formation histories are needed in order to accept or refute the "nature" scenario.

Alternately, processing of PAHs by hard radiation fields has been proposed as a mechanism for the observed correlation between PAH luminosity and metallicity (Madden et al. 2006; Wu et al. 2006; Bendo et al. 2006; Smith et al. 2007; Gordon et al. 2008; Engelbracht et al. 2008), since low-metallicity environments are generally characterized by harder radiation fields than high-metallicity ones (e.g. Hunt et al. 2010). Additionally, efficient destruction of PAHs by supernova shocks may also contribute to the observed deficiency (O'Halloran et al. 2006). These suggestions agree with the observation that PAHs are present in the PDRs surrounding HII regions, but are absent (likely destroyed) within the HII regions (Cesarsky et al. 1996; Helou et al. 2004; Bendo et al. 2006; Relaño \& Kennicutt 2009). A recent study of the SMC finds a high fraction of PAHs within molecular clouds (Sandstrom et al. 2010), thus complicating the interpretation of the formation/destruction mechanisms for these molecules. Both production and processing may ultimately be driving the observed trend (Wu et al. 2006; Engelbracht et al. 2008; Marble et al. 2010).

\section{The heating populations of PAHs}

Outside of HII regions and other strongly ionizing environments, PAHs tend to be ubiquitous. These large molecules are transiently heated by single UV and optical photons, and, therefore, they can be heated by the radiation from the mix of stellar populations that contribute to the general interstellar radiation field, unrelated to the current star formation in a galaxy (Haas et al. 2002; Boselli et al. 2004; Peeters et al. 2004; Mattioda et al. 2005; Calzetti et al. 2007; Draine \& Li 2007; Bendo et al. 2008). 
Bendo et al. (2008) show that the $8 \mu \mathrm{m}$ emission from galaxies is better correlated with the $160 \mu \mathrm{m}$ dust emission than with the $24 \mu \mathrm{m}$ emission, on spatial scales $\approx 2 \mathrm{kpc}$. Haas Klaas \& Bianchi (2002) also find that the ISO $7.7 \mu \mathrm{m}$ emission is correlated with the $850 \mu \mathrm{m}$ emission from galaxies. Boselli et al. (2004) show that the stellar populations responsible for the heating of the MIR-emitting dust are more similar to those responsible for heating the large grains emitting in the far-infrared, rather than to those responsible for the ionized gas emission in galaxies. This result is similar to that obtained by Peeters et al. (2004) for a combined Galactic and extragalactic sample of PAH-emitting sources. All the evidence points to a close relation between the MIR/PAH emission and the cold dust heated by the general (non-star-forming) stellar population.

The presence of heating by evolved stellar populations thus produces a illquantified contribution to the total MIR luminosity of a galaxy, affecting the calibration of any SFR indicator using that wavelength range (e.g., Alonso-Herrero et al. 2006). We still do not have a clear understanding of dependencies on galaxy morphology, stellar population mix, star formation rate, star formation intensity, etc.

A recent analysis of the nearby galaxy NGC 0628, an almost face-on SAc at a distance of about 7.3 Mpc, shows that its $8 \mu \mathrm{m}$ emission contains about $20 \%-30 \%$ contribution from a diffuse component unrelated to sites of current star formation (Crocker et al. 2010). The 20\%-30\% range reflects uncertainties in the adopted extinction and [NII] contamination corrections for the $\mathrm{H} \alpha$ emission, used here to trace sites of recent star formation. We can also obtain a rough idea of the amount of diffuse emission in the MIR bands by converting the $8 \mu$ m mean luminosity density, $\sim 1.2 \times 10^{7} L_{\odot} \mathrm{Mpc}^{-3}$, within the local $10 \mathrm{Mpc}$ (including emission from both PAHs and dust continuum, see Marble et al. 2010) to a volume SFR density, using metallicity-dependent linear calibrations to $\operatorname{SFR}(8 \mu \mathrm{m})$ from the data of Calzetti et al. (2007). The result, $\rho_{\mathrm{SFR}}(8 \mu \mathrm{m}) \sim 0.019 M_{\odot} \mathrm{yr}^{-1} \mathrm{Mpc}^{-3}$, is roughly $30 \%-60 \%$ higher than the commonly accepted values for the SFR density in the local Volume (see references in Hopkins \& Beacom 2006).

While the above suggests that the contribution to the $8 \mu \mathrm{m}$ emission from dust heated by the diffuse stellar populations is not large (less than a factor $\approx 1.5-2$ ), when galaxies are considered as a whole or as populations, we should recall that we don't have a handle on galaxy-to-galaxy variations. Even worse, the diffuse population heating could become a prevalent contribution to the $8 \mu \mathrm{m}$ emission in some galactic regions, thus potentially affecting any investigation of spatially resolved features within galaxies.

\section{Summary and conclusions}

Over the past decade, the accumulation of both spectroscopy and high-angularresolution imaging of the MIR emission from galaxies has paved the road for an accurate investigation of the MIR/PAH luminosity as a SFR tracer. Data have shown that there is generally a linear (with slope of 1 or slightly less than 1 in a loglog plane) correlation between the stellar-continuum-subtracted MIR luminosity 
and the SFR, for high metallicity systems, i.e., for galaxies and regions that are about $1 / 5-1 / 3$ solar or higher in oxygen abundance. While a linear correlation would generally indicate that the MIR emission can be considered a reliable SFR indicator, there are at least two caveats to keep in mind: (1) the PAH emission has a strong dependence on metallicity; (2) the MIR-emitting dust can be heated by evolved stellar populations unrelated to the current star formation.

The PAH dependence on metallicity is well established and quantified, and shows an order-of-magnitude deficiency in PAH/TIR emission for a decrease in oxygen abundance by about a factor 10. The nature of this dependency is still debated, and could be due to delayed production of the PAHs in low metallicity systems or to processing/destruction mechanisms in the harder radiation fields of low metallicity galaxies, or a combination of both.

The heating of PAHs by evolved stellar populations is also well established, but less well quantified. It is likely to have a smaller impact on any SFR(PAH) or SFR(MIR) calibration than the metallicity dependence, probably at the level of less than a factor 2. However, potential variations as a function of galaxy morphology, stellar population mix, star formation rate, star formation intensity, etc., and the impact of the diffuse population heating as a function of location for sub-galactic scale analyses have not been quantified yet. This is a virtually unexplored realm with a possibility for major implications as many investigations move from the "global populations" approach to the "sub-kpc regions" approach in the study of star formation in galaxies.

\section{References}

Alonso-Herrero, A., Colina, L., Packam, C., et al., 2006, ApJ, 652, L83

Aplund, M., Grevesse, N., Sauval, A.J., \& Scott, P., 2009, ARA\&A, 47, 481

Bendo, G.J., Dale, D.A., Draine, B.T., et al., 2006, ApJ, 652, 283

Bendo, G.J., Draine, B.T., Engelbracht, C.W., et al., 2008, MNRAS, 389, 629

Boselli, A., Lequeux, J., \& Gavazzi, G., 2004, A\&A, 428, 409

Calzetti, D., 2001, PASP, 113, 1449

Calzetti, D., Kennicutt, R.C., Bianchi, L., et al., 2005, ApJ, 633, 871

Calzetti, D., Kennicutt, R.C., Engelbracht, C.W., et al., 2007, ApJ, 666, 870

Cesarsky, D., Lequeux, J., Abergel, A., et al., 1996, A\&A, 315, L309

Crocker, A.F., Calzetti, D., et al., 2010, in preparation

Daddi, E., Dickinson, M., Chary, R., et al., 2005, ApJ, 631, L13

Daddi, E., Dickinson, M., Morrison, G., et al., 2007, ApJ, 670, 156

Dale, D.A., Smith, J.T.D., Schlawin, E.A., et al., 2009, ApJ, 693, 1821

Dole, H., Lagache, G., Puget, J.-L., et al., 2006, A\&A, 451, 417

Draine, B.T., \& Li, A., 2007, ApJ, 657, 810

Draine, B.T., Dale, D.A., Bendo, G., et al., 2007, ApJ, 633, 866

Engelbracht, C.W., Gordon, K.D., Rieke, G.H., et al., 2005, ApJ, 628, 29

Engelbracht, C.W., Rieke, G.H., Gordon, K.D., et al., 2008, ApJ, 685, 678

Fazio, G.G., Hora, J.L., Allen, L.E., et al., 2004, ApJS, 154, 10 
Förster Schreiber, N.M., Roussel, H., Sauvage, M., \& Charmandaris, V., 2004, A\&A, 419,501

Galliano, F., Madden, S.C., Jones, A.P., Wilson, C.D., \& Bernard, J.-P., 2005, A\&A, 434,867

Galliano, F., Dwek, E., \& Chanial, P., 2008, ApJ, 672, 214

Gordon, K.D., Engelbracht, C.W., Rieke, G.H., et al., 2008, ApJ, 682, 336

Haas, M., Klaas, U., \& Bianchi, S., 2002, A\&A, 385, L23

Hauser, M.G., \& Dwek, E., 2001, ARA\&A, 39, 249

Heckman, T.M., Robert, C., Leitherer, C., Garnett, D.R., \& van der Rydt, F., 1998, ApJ, 503, 646

Helou, G., Roussel, H., Appleton, P., et al., 2004, ApJS, 154, 253

Hogg, D.W., Tremonti, C.A., Blanton, M.R., et al., 2005, ApJ, 624, 162

Hopkins, A.M., \& Beacom, J.F., 2006, ApJ, 651, 142

Houck, J.R., Roellig, T.L., van Cleve, J., et al., 2004, ApJS, 154, 18

Hunt, L.K., Thuan, T.X., Izotov, Y.I., \& Sauvage, M., 2010, ApJ, 712, 164

Kennicutt, R.C., Hao, C.-N., Calzetti, D., et al., 2009, ApJ, 703, 1672

Kessler, M.F., Steinz, J.A., Anderegg, M.E., et al., 1996, A\&A, 315, L27

Lawton, B., Gordon, K.D., Babler, B., et al., 2010, ApJ, 716, 453

Lee, Janice, C., Kennicutt, R.C., Funes, J.G., Sakai, S., \& Akiyama, S., 2007, ApJ, 671, L113

Léger, A., \& Puget, J.L., 1984, A\&A, 137, L5

Madden, S.C., 2000, in Massive Stellar Clusters, ed. A Lançon, \& C. Boily, ASP Conf. Ser., 211, 297

Madden, S.C., Galliano, F., Jones, A.P., \& Sauvage, M., 2006, A\&A, 446, 877

Marble, A.R., Engelbracht, C.W., van Zee, L., et al., 2010, ApJ, 715, 506

Mattioda, A.L., Allamandola, L.J., \& Hudgins, D.M., 2005, ApJ, 629, 1183

Muñoz-Mateos, J.C., Gil de Paz, A., Boissier, S., et al., 2009, ApJ, 701, 1965

O'Halloran, B., Satyapal, S., \& Dudik, R.P., 2006, ApJ, 641, 795

Papovich, C., Moustakas, L.A., Dickinson, M., et al., 2006, ApJ, 640, 92

Papovich, C., Rudnick, G., Le Floc'h, E., et al., 2007, ApJ, 668, 45

Peeters, E., Spoon, H.W.W., \& Tielens, A.G.G.M., 2004, ApJ, 613, 986

Puget, J.L., Léger, A., \& Boulanger, F., 1985, A\&A, 142, L19

Reddy, N.A., Erb, D.K., Pettini, M., Steidel, C.C., \& Shapley, A.E., 2010, ApJ, 712, 1070

Relaño, M., \& Kennicutt, R.C., 2009, ApJ, 699, 1125

Rieke, G.H., Young, E.T., Engelbracht, C.W., et al., 2004, ApJS, 154, 25

Romano, D., Tosi, M., \& Matteucci, F., 2006, MNRAS, 365, 759

Rosenberg, J.L., Ashby, M.L.N., Salzer, J.J., \& Huang, J.-S., 2006, ApJ, 636, 742

Roussel, H., Sauvage, M., Vigroux, L., \& Bosma, A., 2001, A\&A, 372, 427

Salim, S., Dickinson, M., Rich, R.M., et al., 2009, ApJ, 700, 161

Sandstrom, K.M., Bolatto, A.D., Draine, B.T., Bot, C., \& Staminirović, S., 2010, ApJ, 715,701

Sellgren, K., Werner, M.W., \& Dinerstein, H.L., 1983, ApJ, 271, L13 
Sellgren, K., 1984, ApJ, 277, 623

Smith, J.D.T., Draine, B.T., Dale, D.A., et al., 2007, ApJ, 656, 770

Sullivan, M., Mobasher, B., Chan, B., et al., 2001, ApJ, 558, 72

Tosi, M., 2009, A\&A, 500, 157

Wang, B., \& Heckman, T.M., 1996, ApJ, 457, 645

Werner, M.W., Roellig, T.L., Low, F.J., et al., 2004, ApJS, 154, 1

Wu, H., Cao, C., Hao, C.-N., et al., 2005, ApJ, 632, L79

Wu, Y., Charmandaris, V., Hao, L., et al., 2006, ApJ, 639, 157

Yan, L., Sajina, A., Fadda, D., et al., 2007, ApJ, 658, 778

Zhu, Y.-N., Wu, H., Cao, C., \& Li, H.-N., 2008, ApJ, 686, 155 